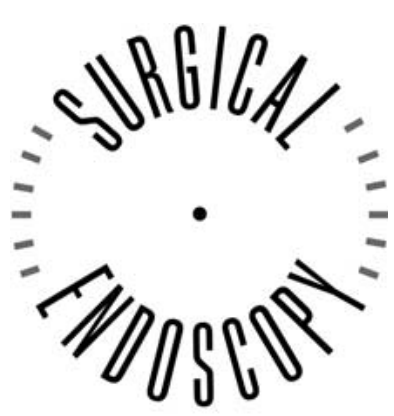

and Other Interventional Techniques

\title{
Recurrence after laparoscopic and open Nissen fundoplication
}

\author{
A comparison of the mechanisms of failure
}

\author{
K. Graziano, D. H. Teitelbaum, K. McLean, R. B. Hirschl, A. G. Coran, J. D. Geiger
}

Department of Surgery, Section of Pediatric Surgery, the C. S. Mott Children's Hospital, University of Michigan, F3970, Box 0245, Ann Arbor, MI 48109, USA

Received: 29 October 2002/Accepted: 5 November 2002/Online publication: 7 March 2003

\begin{abstract}
Background: Laparoscopic Nissen fundoplication as treatment for gastroesophageal reflux disease (GERD) in adults has a reported recurrence rate of $2-17 \%$. We investigated the rates and mechanisms of failure after laparoscopic Nissen fundoplication in children.

Methods: All patients who underwent a laparoscopic Nissen fundoplication for GERD and who subsequently required a redo Nissen were reviewed $(n=15)$. The control group consisted of the most recent 15 patients who developed recurrent GER after an open Nissen, fundoplication.

Results: Between 1994 and 2000, laparoscopic Nissen fundoplication was performed in 179 patients. Fifteen patients $(8.7 \%)$ underwent revision. The mechanisms of failure were herniation in four patients, wrap dehiscence in four, a too-short wrap in three, a loosened wrap in two, and other reasons in two. The reoperation was performed laparoscopically in five patients (33\%). The failure mechanisms were different in the open patients: eight were due to slipped wraps; three to dehiscences; and two to herniations.

Conclusion: The failure rate after laparoscopic Nissen is acceptably low. A redo laparoscopic Nissen can be performed safely after an initial laparoscopic approach.
\end{abstract}

Key words: Gastroesophageal reflux disease (GERD) - Nissen fundoplication - Laparoscopic surgery Reoperation - Children

The failure rate after open Nissen fundoplication in children has been reported to range from 2 to $24 \%[1,3$, $5,8,13]$. However, the failure rate after the comparable laparoscopic procedure has not been studied in children. In the adult literature, the reported rate of laparoscopic failure after antireflux procedures is $2-17 \%$ - comparable to, if not slightly better than, the open failure rate in

Correspondence to: J. D. Geiger adults of 9-30\% [7]. The purpose of this study was to evaluate the failure rate after laparoscopic Nissen fundoplication for gastroesophageal reflux disease (GERD) in children as compared to the failure rate after open Nissen fundoplication. We also sought to investigate the mechanisms of failure in the two groups and to examine our early experience with the laparoscopic approach for reoperation after the failure of a Nissen procedure.

\section{Patients and methods}

Between 1994 and 2000, we performed laparoscopic Nissen fundoplication for GERD in 179 patients. Fifteen patients who underwent a laparoscopic Nissen fundoplication between January 1996 and October 1999 and subsequently required a redo Nissen were identified. For comparison, a cohort of the most recent 15 patients who initially underwent an open Nissen fundoplication and subsequently required reoperation were also identified. Documents, including operative summaries, clinic notes, and radiologic reports, were analyzed retrospectively for age at initial operation, age at reoperation, other procedures performed at the time of fundoplication (e.g., gastrostomy tube placement, pyloroplasty, pyloromyotomy), indications for reoperation, radiological findings, operative findings, and coexisting diagnoses.

The diagnosis of recurrence, in addition to symptoms, was diagnosed objectively with an upper gastrointestinal contrast study documenting anatomical recurrence and/or reflux, a $\mathrm{pH}$ probe study documenting reflux, or both. All patients underwent a Nissen fundoplication, either via the laparoscopic or the open approach. A short (2.0-cm or three-suture fundoplication), loose (sized over an appropriate bougie) wrap was the standard approach. The short gastric vessels were divided, and a crural repair sized appropriately with a bougie was performed in all patients.

Data were analyzed using Student's $t$-test; statistical significance was assigned at $p<0.05$. Variables are expressed as mean \pm standard deviation (SD).

\section{Results}

Fifteen of 179 patients who underwent laparoscopic Nissen fundoplication at our institution required reop- 
Table 1. Group characteristics and reoperative approaches

\begin{tabular}{lcc}
\hline & $\begin{array}{l}\text { Laparoscopic } \\
\text { group }\end{array}$ & $\begin{array}{l}\text { Open } \\
\text { group }\end{array}$ \\
\hline Female patients & 4 & 8 \\
Male patients & 11 & 7 \\
Age at first operation (mo) & 28 & 44 \\
Age at reoperation (mo) & 39 & 76 \\
Interim to reoperation (mo) & 11 & 32 \\
Reoperative approach & 5 & 2 \\
$\quad$ Laparoscopic & 6 & 13 \\
Open & 4 & 0 \\
Conversion to open & & 2 \\
Additional procedures at first operation & 7 & 2 \\
$\quad$ Gastrostomy & 0 & 1 \\
Pyloromyotomy & 0 & 3 \\
$\quad$ Pyloroplasty & 1 & 1 \\
Combination of above & & 5 \\
Additional procedures second operation & 2 & 4 \\
$\quad$ Gastrostomy & 4 & 1 \\
$\quad$ Pyloromyotomy & 1 & \\
Pyloroplasty & 2 & \\
$\quad$ Combination of above & & \\
\hline
\end{tabular}

eration; thus, the failure rate was $8.7 \%$. By comparison, our previously reported open Nissen failure rate was $12 \%$ [13]. The characteristics of the laparoscopic group and the open control group are shown in Table 1. There were more boys in the laparoscopic group (73\%) than the open group (47\%). The average age at first wrap tended to be younger in the laparoscopic group than the open group ( 28 vs 44 months), although this finding was not statistically significant $(p=0.37)$. The age at reoperation was 39 months in the laparoscopic group vs 76 months in the open group, and the interval between operations averaged 11 months in the laparoscopic group vs 32 months in the open group. This shorter interval in the laparoscopic group was statistically different $(p=0.01)$. Nine of the patients in the laparoscopic group $(60 \%)$ and 11 in the open group (73\%) underwent an additional procedure at the time of initial wrap, including placement of a gastrostomy tube, pyloromyotomy, pyloroplasty, or a combination of two of these procedures. At reoperation, nine of the patients in the laparoscopic group and 11 of the patients in the open group underwent another related procedure. In both groups, more patients underwent a pyloromyotomy at reoperation than at the initial operation.

An attempt to follow the laparoscopic approach during the reoperation was made in nine of the 15 patients who had initially undergone a laparoscopic procedures. Five of these attempts were completed successfully, and four patients were converted to open procedures. Reasons for conversion included dense adhesions between the stomach and liver and other surrounding structures $(n=2)$, dense adhesions at the gastroesophageal junction precluding adequate visualization of the wrap $(n=1)$, and an esophagotomy during dissection of the wrap requiring open repair $(n=1)$. Of the 15 patients who had originally undergone an open Nissen fundoplication, two patients had a successful laparoscopic revision and the other 13 had an open reoperation.

After the initial wrap, patients presented with a variety of symptoms that suggested reflux recurrence or

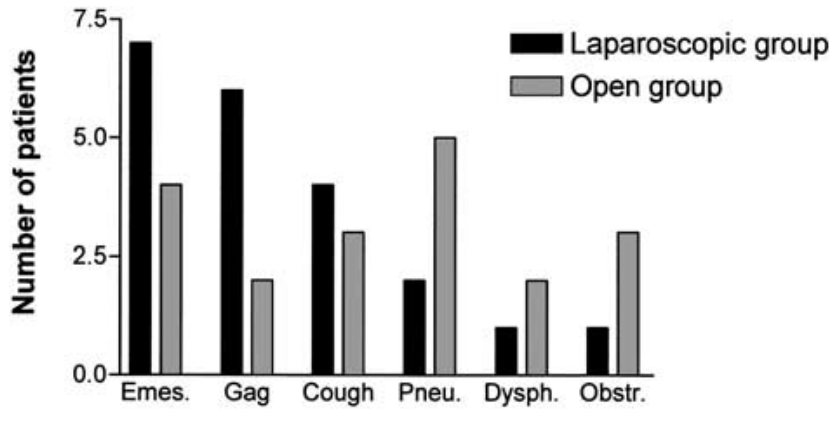

Symptoms

Fig. 1. Indications for reoperation after laparoscopic and open Nissen fundoplication. Lmes, emesis; Egg, gogging; Cough, coughing; Pheu, Pneumonia; Dysph, dysphagia; Obst, obstruction.

some other failure of the fundoplication. Many of the patients had more than one symptom. The frequency of symptoms in the two groups is shown in Fig. 1. In the laparoscopic group, seven patients experienced frequent emesis $(47 \%)$, six experienced gagging $(40 \%)$, four had frequent coughing episodes $(27 \%)$, two had pneumonia requiring hospitalization (13\%), one patient had dysphagia, and one patient had obstructive symptoms (7\%). In the group that initially underwent an open approach, five patients developed pneumonia requiring hospitalization (33\%), four experienced frequent emesis (27\%), three experienced coughing episodes $(20 \%)$, three patients had obstructive symptoms (20\%), two patients had gagging, and two had dysphagia (13\%).

All patients underwent an upper gastrointestinal contrast study prior to reoperation. The radiologic findings were then correlated with the operative findings to determine the mechanism of wrap failure. In the laparoscopic group, the mechanisms of failure were as follows: four patients had a transdiaphragmatic herniation of the wrap (27\%), four patients had a partial wrap dehiscence $(27 \%)$, three patients had a short $(<1 \mathrm{~cm})$ wrap that allowed for recurrent reflux $(20 \%)$, and two patients had apparently loosened wraps (13\%). In two other patients, other mechanisms led to the failure; one patient developed a stricture at the gastroesophageal junction, and in the other case the fundus of the stomach had folded due to adhesions, causing obstructive symptoms (Fig. 2).

The mechanisms of failure in the open group included slippage of the wrap onto the fundus in eight patients (53\%), partial or complete dehiscence of the wrap in three patients (20\%), and transdiaphragmatic herniation of the wrap in two patients (13\%). Other mechanisms in two patients included a loosened wrap in one case and adhesion to the liver causing twisting of the fundus and obstructive symptoms in another (Fig. 3).

Of the 15 upper gastrointestinal contrast studies done in the laparoscopic group, 11 were positive for reflux (73\%), two showed transdiaphragmatic herniation of the wrap, and two were negative for recurrent reflux or any mechanical abnormalities. Of the 15 studies done in the open group, eight studies were positive for reflux $(53 \%)$, and one study showed a dysmotile esophagus; the remaining six patients had studies that were negative for 


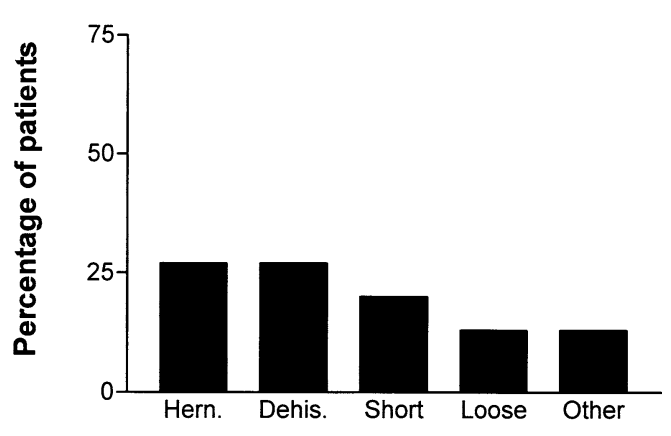

Mechanism

Fig. 2. Mechanisms of failure after laparoscopic Nissen fundoplication. Hern, herniation; Dehis, dehiscence.

recurrent reflux or mechanical abnormalities. In five of the laparoscopic patients and seven of the open patients, 24-h pH probes were placed to further document recurrent reflux. In all cases, these studies were positive for reflux, including the studies in patients who had previously had upper gastrointestinal contrast studies that did not document reflux.

Both groups were similar in terms of the presence of other diagnoses. In the laparoscopic group, 12 of the 15 patients had coexisting diagnoses, including six patients (40\%) who were neurologically impaired. In the open group, 13 of the 15 patients had coexisting diagnoses, and nine of them $(60 \%)$ had some neurological impairment. Four of the patients in the open group required a third operation to treat their recurrent reflux symptoms, and in one case a fourth operation was needed. None of the patients in the laparoscopic group required a third operation.

\section{Discussion}

There have been several studies documenting the failure rate after open surgery for GERD in children. In one study evaluating complications and the need for reoperation in 429 infants and children undergoing Nissen fundoplication, 38 children required a second fundoplication, for a reoperation rate of $8.8 \%$ [3]. Another study that evaluated 66 patients requiring redo fundoplication over a 15 -year period reported a failure rate of $9 \%$ [8]. In a combined hospital study of 7,467 patients, the reoperation rates ranged from $2 \%$ to $24 \%$ [5]. A fourth study of 364 patients reported a reoperation rate of $6 \%$ [1]. Studies in adults have found similar rates of failure for open fundoplication, ranging from $9 \%$ to $30 \%$ [7].

However, rates of failure after laparoscopic fundoplication, have not been as well documented. In adults, the failure rate after laparoscopic Nissen ranges from $2 \%$ to $17 \%$, depending on the length of follow-up [7]. In children, the rate of failure after laparoscopic Nissen fundoplication is not known. In the multicenter study of 7,467 patients, $2.6 \%$ of patients underwent a laparoscopic Nissen fundoplication, but the failure rate in this subset of patients was not evaluated [5]. Our failure rate of $8.7 \%$ after laparoscopic Nissen fundoplication is comparable to the rates of failure after the comparable open procedure in children.

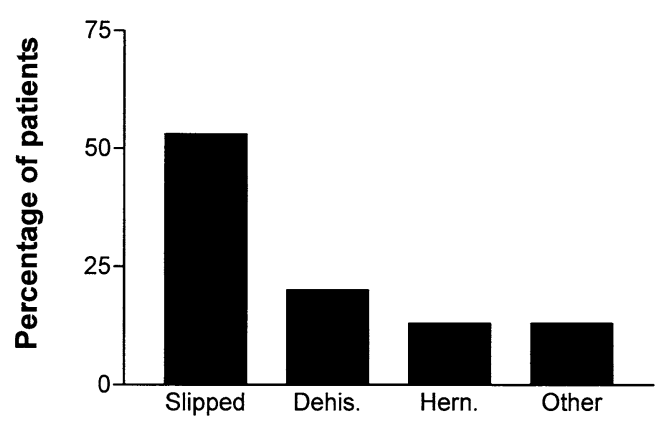

\section{Mechanism}

Fig. 3. Mechanisms of failure after open Nissen fundoplication. Dehis, dehiscence; Hern, herniotion.

The mechanisms of failure after open fundoplication in children have been well defined. Four main mechanisms have been described: slipped or misplaced fundoplication, disrupted fundoplication, herniated fundoplication, and a fundoplication that is too tight or too long $[1-3,8]$. In one study, 28 of 429 patients $(6.7 \%)$ experienced a breakdown or herniation of the wrap after the initial procedure, six patients (1.4\%) developed a stricture, and four patients $(0.9 \%)$ had a partial wrap breakdown [3]. The same institution later published results from a different group of 130 patients undergoing reoperation after Nissen fundoplication and found the mechanisms of failure to be as follows: wrap breakdown (42\%), transdiaphragmatic herniation (30\%), or both of these mechanisms (21\%) [2]. Kimber et al.'s study of the mechanisms of failure in 66 patients, 30 patients had transdiaphragmatic herniation $(45 \%), 10$ patients $(15 \%)$ had dehiscence, 22 patients $(33 \%)$ had a combination of these, and four patients $(6 \%)$ had a wrap that was too tight [8]. A study by Caniano et al. of reoperations in 21 children revealed slippage of the wrap in 15 patients (71\%), dehiscence in two patients $(9.5 \%)$, and transdiaphragmatic herniation in two patients $(9.5 \%)$ [1]. Our own results after the open Nissen procedure included eight patients with slippage of the wrap onto the fundus of the stomach, a finding similar to the results of $\mathrm{Ca}$ niano et al. This notable difference between these two studies and the other published series may reflect a difference in the way the mechanisms of failure are defined. "Wrap disruption" as described in the other studies may have included slipped or misplaced fundoplications.

The mechanisms of failure after laparoscopic Nissen fundoplication in children have not been studied. A study in adults that included 31 patients undergoing reoperation after a laparoscopic Nissen procedure reported that 13 patients had suffered a transdiaphragmatic herniation of the wrap (43\%) [3]. In another study reviewing 720 patients undergoing laparoscopic Nissen fundoplication, six patients required reoperation for paraesophageal herniation [8]. In the largest reported study of adult patients undergoing a laparoscopic Nissen procedure, 31 of 857 patients required reoperation; 26 of these cases, the mechanism of failure was transdiaphragmatic herniation (84\%) [7]. These studies identified two additional failure mechanisms: the twisted fundoplication and the two-compartment stomach. The 
Table 2. Success rates of laparoscopic reoperation in adults

\begin{tabular}{llc}
\hline Series [ref. no.] & $\begin{array}{l}\text { No. of } \\
\text { patients }\end{array}$ & $\begin{array}{l}\text { Lap redo } \\
(n, \%)\end{array}$ \\
\hline $\begin{array}{l}\text { Watson et al. [12] } \\
\text { Laparoscopic }\end{array}$ & 14 & $12(86)$ \\
$\quad$ Open & 13 & $9(69)$ \\
$\begin{array}{l}\text { Floch et al. [4] } \\
\text { Laparoscopic }\end{array}$ & 22 & $20(91)$ \\
$\quad$ Open & 24 & $17(71)$ \\
Pointner et al. [10] & & $12(100)$ \\
$\quad$ Laparoscopic & 12 & $16(89)$ \\
$\quad$ Open & 18 & \\
\hline
\end{tabular}

mechanisms of failure in our small group of 15 children included fewer herniations $(27 \%)$ than expected when compared to the studies in adults. However, we are following a number of patients with partially herniated fundoplications whose symptoms are not severe enough to warrant reoperation.

In our series, the interim between the initial operation and the reoperation differed between the laparoscopic group and the open group: The open group had the second operation 32 months later, whereas the laparoscopic group was going reoperated after only 11 months. In Dedinsky et al.'s series of 38 reoperations in children after open Nissen fundoplication, the interim to reoperation averaged 20 months [3]. The series of 130 patients reported by Dalla Vecchia et al. found reported the interim to reoperation to be on average 21 months [2]. There are no data evaluating reoperation after laparoscopic Nissen in children, and it is unclear why our patients presented earlier than those reported in the studies on open Nissen fundoplication. In adults requiring reoperation after laparoscopic Nissen, 50\% undergo revision within 12 months of the initial operation and $90 \%$ undergo revision within 24 months of the initial operation [7].

In our series, laparoscopic reoperation was performed successfully in $23 \%$ of the 30 children who required revision, regardless of the approach used for the initial operation. We performed laparoscopic revision in $33 \%$ of the patients who had initially undergone a laparoscopic approach for their first fundoplication. The effectiveness of laparoscopic reoperation after failed Nissen has recently been well documented in the adult literature. Table 2 lists the success rates for redo laparoscopic Nissen after open and laparoscopic wraps in three series of adults. The success rates were quite good - in the range of $69-100 \%[1,2,4-6,10-13]$. The success rates for redo laparoscopic surgery after an initial laparoscopic approach ranged from $86 \%$ to $100 \%$ in these series. After our early attempts at laparoscopic reoperation in patients who had undergone either an open or a laparoscopic fundoplication, we conclude that the laparoscopic procedure is feasible and can be safely completed. It is too early in our learning curve to definitively judge redo laparoscopic fundoplication.

Some of the series of reoperations after open fundoplication in children have shown differences between patients who are neurologically normal and those who are neurologically impaired. In one study, only four of 81 neurologically normal patients required reopera- tion (5\%) as compared to 29 of 153 neurologically impaired patients (19\%) [1]. Moreover, the study found a significant difference in the number of neurologically impaired patients who required reoperation due to transdiaphragmatic herniation. In Dalla Vecchia et al.'s study of 130 reoperations, 101 patients $(78 \%)$ were neurologically impaired [2]. Many reasons have been suggested for this increased risk of failure in neurologically impaired patients, but there are no firm data to support any single reason. Aggressive control of the medical conditions that contribute to recurrence, including cerebral palsy, seizure disorder, and chronic pulmonary disease, has been recommended as a measure to prevent mechanical disruption of the wrap [2,3].

Our failure rate after laparoscopic Nissen of $8.7 \%$ is acceptably low and compares well to failure rates in children after open Nissen fundoplication. The mechanisms of failure after laparoscopic Nissen fundoplication appear to be different from open fundoplication, with increased wrap herniation and dehiscence. In an attempt to reduce the incidence of these mechanisms of failure, we have employed the following modifications of the laparoscopic technique: (a) minimization of hiatal dissection, (b) meticulous crural repair, (c) fixation of the wrap to the diaphragm, and (d) avoidance of early postoperative retching and gagging.

\section{References}

1. Caniano DA, Ginn-Pease ME, King DR (1990) The failed antireflux procedure: analysis of risk factors and morbidity. J Pediatr Surg 25: 1022-1026

2. Dalla Vecchia LK, Grosfeld JL, West KW, Rescorla FJ, Scherer LR, Engum SA (1997) Reoperation after Nissen fundoplication in children with gastroesophageal reflux: experience with 130 patients. Ann Surg 226:

3. Dedinsky GK, Vane DW, Black CT, Turner MK, West KW, Grosfeld JL (1987) Complications and reoperation after Nissen fundoplication in childhood. Am J Surg 153: 177-183

4. Floch NR, Hinder RA, Klinger PJ, et al. (1999) Is laparoscopic reoperation for failed antireflux surgery feasible? Arch Surg 134: 733-737

5. Fonkulsrud EW, Ashcraft KW, Coran AG, et al. (1998) Surgical treatment of gastroesophageal reflux in children: a combined hospital study of 7467 patients. Pediatrics 101: 419-422

6. Horgan S, Pohl D, Bogetti D, et al. (1999) Failed antireflux surgery: what have we learned from reoperations? Arch Surg 134: 809-817

7. Hunter JG, Smith CD, Branum GD, Waring JP, Trus TL, Cornwell M, Galloway K (1999) Laparoscopic fundoplication failures: patterns of failure and response to fundoplication revision. Ann Surg 230:

8. Kimber C, Kiely EM, Spitz L (1998) The failure rate of surgery for gastro-oesophageal reflux. J Pediatr Surg 33: 64-66

9. Pearl RH, Robie DK, Ein SH, et al. (1990) Complications of gastroesophageal antireflux surgery in neurologically impaired versus neurologically normal children. J Pediatr Surg 25: 1169-1173

10. Pointner R, Bammer T, Then P, et al. (1999) Laparoscopic refundoplications after failed antireflux surgery. Am J Surg 178: 541-544

11. Seelig MH, Hinder RA, Klinger PJ, et al. (1999) Paraesophageal herniation as a complication following laparoscopic antireflux surgery. J Gastrointest Surg 3: 95-99

12. Watson DI, Jamieson GG, Game PA, et al. (1999) Laparoscopic reoperation following failed antireflux surgery. Br J Surg 86: 98-101

13. Wheatley MJ, Coran AG, Wesley JR, et al. (1991) Redo fundoplication in infants and children with recurrent gastroesophageal reflux. J Pediatr Surg 26: 758-761 